\title{
RESPONSIVE POLYMER HYBRID GEL CROSS-LINKED BY N,N-(1,2-DIHYDROXYETHYLENE) BISACRYLAMIDE FOR CATALYTIC APPLICATION
}

\author{
${ }^{1}$ ZAFFAR IQBAL,${ }^{1,2}$ LUQMAN ALI SHAH $*,{ }^{3}$ MURTAZA SAYED ${ }^{1}$ ABDUL HALEEM AND \\ ${ }^{1} M O H A M M A D$ SIDDIQ*
}

\author{
${ }^{I}$ Department of Chemistry, Quaid-I-Azam University, Islamabad 45320, Pakistan \\ ${ }^{2}$ National Centre of Excellence in Physical Chemistry, University of Peshawar, Peshawar 25120 Pakistan \\ ${ }^{3}$ Department of Chemistry, COMSATS Institute of Information technology, 22060, Abbottabad, Pakistan
}

\begin{abstract}
Copolymer microgel composed of $\mathrm{N}$-isopropyl acrylamide and methacrylic acid, poly(NIPAM-MAA), was synthesized by free radical emulsion polymerization using N,N-(1,2-dihydroxyethylene) bisacrylamide as cross-linking agent. The hybrid microgels poly(NIPAM-MAA)-Au and poly(NIPAM-MAA)-Ag, were synthesized by in-situ reduction of metal ions using sodium borohydride $\left(\mathrm{NaBH}_{4}\right)$ as reducing agent. The temperature and $\mathrm{pH}$ sensitivity of pure and hybrid microgels were studied by dynamic light scattering (DLS) and UV-Visible spectroscopy. The existence of gold and silver nanoparticles inside the microgel network was confirmed by transmission electron microscopy (TEM). Poly(NIPAM-MAA)-Au and poly(NIPAM-MAA)-Ag hybrid microgels were tested for the comparative catalytic activity towards the reduction of 4-nitrophenol to 4-aminophenol. The apparent rate constant ( $\left.k_{\text {app }}\right)$ for poly(NIPAM-MAA)-Au was found superior than poly(NIPAM-MAA)-Ag.
\end{abstract}

Keywords: Free radical emulsion polymerization, in-situ reduction method, hybrid microgels, surface plasmon resonance, catalytic activity

\subsection{INTRODUCTION}

Due to enhanced physical, chemical and biological properties, noble metal nanoparticles (NP's) have drawn a considerable attention in the field of energy conversion ${ }^{1}$, drug delivery ${ }^{2}$, sensors ${ }^{3}$ and especially in catalysis ${ }^{4}$. A number of studies have shown that metal nanoparticles (NP's) exhibit unexpectedly high catalytic activities toward different types of reactions due to their high surface area to volume ratio ${ }^{5}$. However, the enhanced surface energy causes aggregation of these NP's and hinders their applications in many fields.

In recent decades, many attempts have been made to overcome this problem. It has been proven by various studies that anchoring metal NP's on supports is an effective approach to solve the aggregation problem. Therefore, many materials have been designed for the stabilization of metal NP's, such as surfactants ${ }^{6}$, micelles ${ }^{7}$, microspheres ${ }^{8}$, hollow capsules ${ }^{9}$, polymer brushes ${ }^{10}$, polymer microgels ${ }^{11}$ and dendrimers ${ }^{12}$. Of the wide range of materials, polymer microgels have attracted considerable interest due to their high surface area and quick responsive behavior towards environmental stimuli. Agrawal et al ${ }^{13}$ prepared $\mathrm{N}$-vinylcaprolactam/acetoacetoxyethyl methacrylate/acrylic acid microgels for the entrapment of colloidal gold nanostructure and studied their catalytic properties. Zheng et $\mathrm{al}^{14}$ synthesized chitosan and acrylic acid based granular hydrogels for the stabilization of silver nanoparticles and used it for the catalytic reduction of organic dyes. We have successfully fabricated silver nanoparticles inside the pure thermo responsive poly(N-isopropyl acrylamide) microgels synthesized with different crosslinking density ${ }^{15}$ and dual responsive cationic poly( $\mathrm{N}$-isopropyl acrylamide-co-vinylimidazole) microgels ${ }^{16}$. The hybrid microgels were used as a catalyst for the reduction of 4-nitrophenol at entire $\mathrm{pH}$ range and the catalyst was found more efficient at $\mathrm{pH}$ $<6$. In all these studies $\mathrm{N}, \mathrm{N}$-methylene bisacrylamide used as a crosslinking agent. To best of our knowledge very little work has been done using N,N(1,2-Dihydroxyethylene) bisacrylamide (DHEBA) as cross linking moiety ${ }^{17}$ for 3D microgel formation and used as a templet for immobilization of metal nanoparticles.

In this work we have carried out the synthesis of stable poly(NIPAMMAA) microgels has been explored by using DHEBA as cross-linker for the fabrication of silver and gold nanoparticles. The effect of metal nanoparticles incorporation on size of microgels at different temperature and $\mathrm{pH}$ conditions has been studied. The synthesized materials were than tested for their catalytic activity by reducing 4-nitrophenol to 4-aminophenol using $\mathrm{NaBH}_{4}$. In this study the efficiency of poly(NIPAM-MAA)-Au catalyst was found more than poly(NIPAM-MAA)-Ag catalyst.

\subsection{EXPERIMENTAL}

$\mathrm{N}, \mathrm{N}-(1,2-\mathrm{Dihydroxy}$ ethylene) bisacrylamide (DHEBA) and sodium dodecyl sulphate (SDS) were obtained from Fluka, ammonium peroxodisulphate (APS) from Scharlau and all other materials were purchased from Sigma Aldrich.

N-isopropyl acrylamide (NIPAM) was recrystallized from a mixture (1:1 volume ratio) of $n$-hexane and toluene. Methacrylic acid (MAA) was purified from inhibitors by distillation under reduced pressure prior to use. The cross-linker N,N-(1,2-Dihydroxyethylene) bisacrylamide (DHEBA), initiator ammonium peroxodisulphate (APS), emulsifying agent sodium dodecyl sulphate (SDS) and all other chemicals were used as received without further treating and purification.

\subsection{Synthesis of poly(NIPAM-MAA) microgel}

The synthesis of poly(NIPAM-MAA) microgel was carried out by free radical emulsion polymerization. The monomers $\mathrm{N}$-isopropyl acrylamide (NIPAM, 0.952g), methacrylic acid (MAA, 103 $\mu \mathrm{L}$ ), N,N-(1,2Dihydroxyethylene) bisacrylamide (DHEBA, $0.077 \mathrm{~g}$ ) as cross linking monomer and $0.05 \mathrm{~g}$ of sodium dodecyl sulphate (SDS) as an emulsifying agent were mixed in a three necked round bottom flask containing $100 \mathrm{~mL}$ of deionized water by keeping total concentration of mixture 0.01 moles. A constant stirring at $320 \mathrm{rpm}$ was performed during reaction and continues nitrogen purging for 45 minutes to create an inert atmosphere by removing oxygen from reaction mixture. After this, when the reaction temperature was $70^{\circ} \mathrm{C}, 5 \mathrm{~mL}$ of APS solution with concentration $0.05 \mathrm{M}$ was added, with the addition of APS the colourless reaction mixture was changed to milky white stable colloidal solution. The reaction was continued for 6 hours, after completion of reaction the microgel was cooled and purified by dialysis using semi permeable membrane (MWCO:12000-14000) for the removal of SDS, unreacted monomers, initiator molecules etc. The deionised water was replaced twice a day for 14 days.

2.2 Synthesis of poly(NIPAM-MAA)-Au and poly(NIPAM-MAA)-Ag hybrid microgels

Poly(NIPAM-MAA)-Au and poly(NIPAM-MAA)-Ag hybrid microgels were synthesised by in-situ reduction method, the metal ions were reduced inside the polymer microgel network using $\mathrm{NaBH}_{4}$ as reducing agent.

Poly(NIPAM-MAA)-Au hybrid microgel was synthesized by diluting $15 \mathrm{ml}$ of pure microgel to $45 \mathrm{ml}$ with deionized water, $5 \mathrm{ml}$ of $\mathrm{HAuCl}_{4} \cdot 4 \mathrm{H}_{2} \mathrm{O}$ $(1 \mathrm{mM})$ was put in the reaction mixture. The mixture was then stirred for 2 hours with continues $\mathrm{N}_{2}$ purging at room temperature. $5 \mathrm{ml}$ of $1.1 \mathrm{mM}$ solution of $\mathrm{NaBH}_{4}$ was added drop wise very slowly to the mixture; colour change was observed indicating the reduction of metal ions, reaction was kept for 4 hours to complete. The solution was dialyzed against frequently changed deionized water for 2 hours to remove the reduced metal particles outside the polymer network. The same process was followed for the synthesis of poly(NIPAMMAA)-Ag hybrid microgel using $5 \mathrm{ml}$ of $1 \mathrm{mM}$ solution of $\mathrm{AgNO}_{3}$.

2.3 Catalytic activity of hybrid microgels

Catalytic activity was investigated for the synthesised hybrid microgels used as a catalyst for the conversion of 4-nitrophenol to 4-aminophenol by $\mathrm{NaBH}_{4}$ as a model reaction. $80 \mathrm{ml}$ of solution was prepared by dissolving $1.5 \mathrm{mg}$ of 4-nitrophenol and $0.2 \mathrm{~g}$ of sodium borohydride. $0.1 \mathrm{ml}$ hybrid microgel 
having $2 \mu \mathrm{M}$ metal nanoparticles was used as a catalyst.

\subsection{Characterization}

The FT-IR spectra of dried microgels were recorded on a NEXUX-470 spectrometer to identify different functional groups present in the microgels. DLLS experiments were performed with a standard laser light scattering spectrometer (Brookhaven Instruments) at an angle of $90^{\circ}$. He-Ne laser $(35 \mathrm{~mW}$, $637 \mathrm{~nm}$ ) was used as a light source. Before performing measurements all the samples were passed through hydrophilic Millipore Millex -HV filters with a pore size of $0.45 \mu \mathrm{m}$ to remove any dust particles. The UV-Visible absorption spectra were taken with Shimadzu 1601 UV-Vis spectrometer. Transmission electron microscopy (TEM) images were obtained on a JEOL JEM $2100 \mathrm{~F}$ transmission electron microscope, and samples for TEM measurements were made by casting one drop of the sample's solution on a carbon copper grid.

\subsection{RESULTS AND DISCUSSION}

\section{$3.1 \quad$ Fourier Transform Infrared Spectroscopy (FT-IR)}

Polymerization and microgel formation was confirmed by FTIR spectroscopy. The FTIR spectra for pure and hybrid microgels are presented in Fig 1. The strong peaks in the range of 690 to $1000 \mathrm{~cm}^{-1}$ due to vinyl group disappeared, that revealed the polymerization has been taken place. The broadening of $-\mathrm{NH}$ stretching peak at $3305 \mathrm{~cm}^{-1}$ indicates the $\mathrm{H}$-bonding and confirms the formation of gel. The two peaks at 2973 and $2933 \mathrm{~cm}^{-1}$ are for asymmetric and symmetric $-\mathrm{CH}$ stretching respectively. A peak at $1651 \mathrm{~cm}^{-1}$ shows $-\mathrm{C}=\mathrm{O}$ of amide and $1544 \mathrm{~cm}^{-1}$ corresponds to $\mathrm{N}-\mathrm{H}$ bending vibration. $-\mathrm{CH}_{2}$ and $-\mathrm{CH}_{3}$ bending vibrations were also observed at $1459 \mathrm{~cm}^{-1}$ and 1387 $\mathrm{cm}^{-1}$ respectively ${ }^{18}$. The FTIR spectra of hybrid microgels show the same peaks like pure microgels but with the clear shifting of the carbonyl peak at 1651 $\mathrm{cm}^{-1}$ due to the interaction of metal nanoparticles with $-\mathrm{C}=\mathrm{O}$ group. This shift is more pronounced in poly(NIPAM-MAA)-Au compared to poly(NIPAMMAA)-Ag, because of the small size of Au particles compared to Ag.

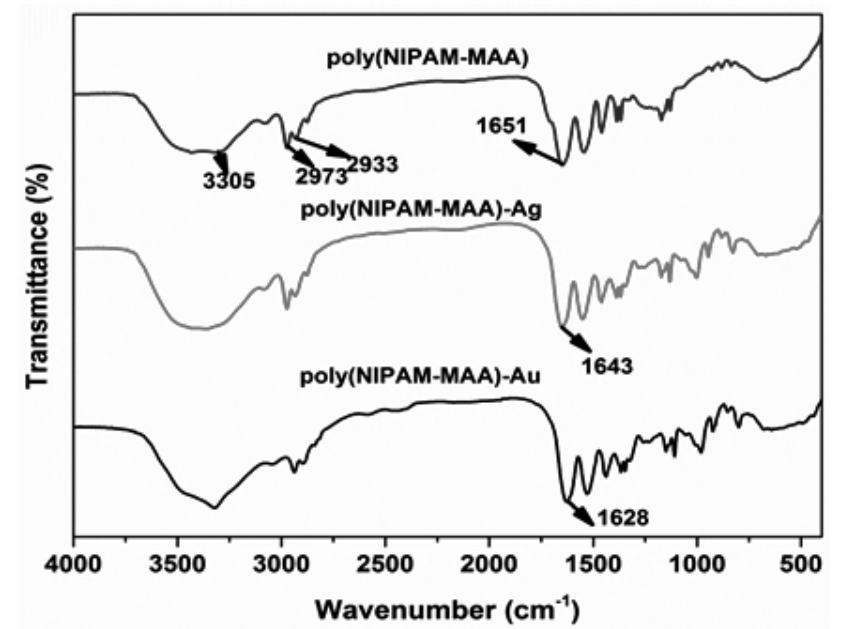

Fig 1 FT-IR spectra of synthesized pure and hybrid microgels

\subsection{Dynamic Laser light scattering (DLLS)}

Dynamic laser light scattering (DLLS) was used for the determination of hydrodynamic diameter of poly(NIPAM-MAA), poly(NIPAM-MAA)-Ag and poly(NIPAM-MAA)-Au microgels at different $\mathrm{pH}$ and temperature. The influence of $\mathrm{pH}$ on the hydrodynamic diameter is given in Fig 2. It was found that the increase in $\mathrm{pH}$ of the medium cause enhancement in size for both pure and hybrid microgel particles. This is due to the deprotonation of carboxyl group in polymer segments. At $\mathrm{pH}$ below $\mathrm{pKa}$ value, the carboxylic group is in protonated form and microgel particles exist in collapsed state. At $\mathrm{pH}$ above pKa value, the carboxylic groups become deprotonated, which produces strong repulsive forces and swelling of the particles takes place. At $\mathrm{pH}$ close to $\mathrm{pKa}$ value, the particles exist in partially protonated form and indicate the transition from collapsed to swollen states. The hydrodynamic diameter of hybrid microgels is lower than pure microgel particles; this is because of two reasons. Firstly, the presence of silver and gold nanoparticles in the polymer network behave as a physical cross-linker which tight the network slightly as compared to pure particles. Secondly, the complexation between metal nanoparticles and polymer chains causes shrinkage in particles. Furthermore the trend of increase in size as a function of $\mathrm{pH}$ was almost same for both pure and hybrid microgels 19. The shrinkage in particle size was found greater for PG-Au compared to PG-Ag, because of the small size of gold nanoparticles and high interaction with polymer chains ${ }^{13,20}$.

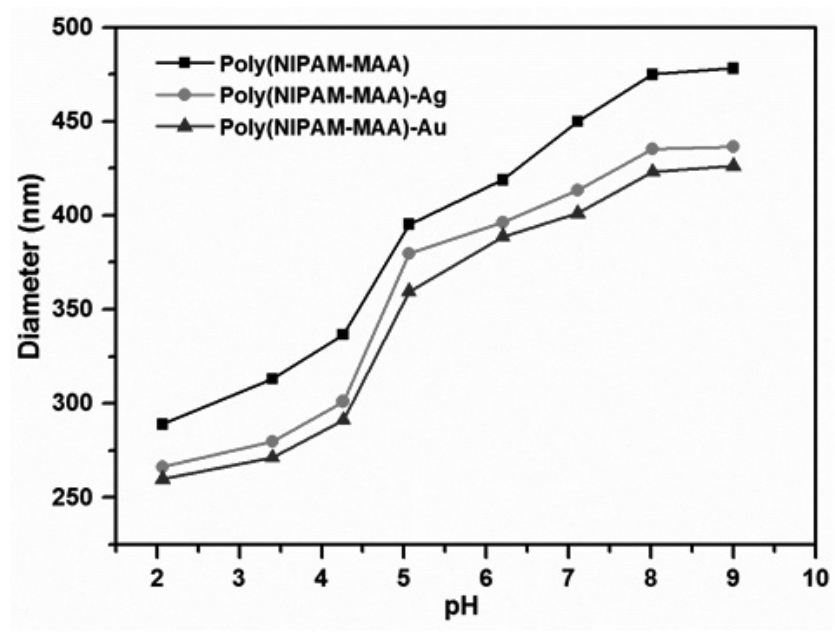

Fig 2 Hydrodynamic diameter as a function of $\mathrm{pH}$

Temperature sensitivity for the pure and hybrid microgels was investigated at $\mathrm{pH} 3.11$ and 8.0. The $\mathrm{pH}$ values were adjusted by using freshly prepared dilute solutions of $\mathrm{HCl}$ and $\mathrm{NaOH}$. At $\mathrm{pH} 3.11$, the particles exist in shrunken state and the temperature effect is more pronounced. The increase in temperature cause a reduction in particle size for both pure and hybrid microgels. At low temperature, water act as a good solvent due to strong hydrophilic interactions between water molecules and hydrophilic part of polymer chains. These interactions enable water molecules to penetrate inside the polymer network and increase the particle size. The increase in temperature causes the disruption of hydrophilic forces and expel water molecules from the polymer network, which causes reduction in particle size. The size of microgel shows a rapid decrease at $30^{\circ} \mathrm{C}$ which corresponds to the volume phase transition temperature (VPTT), after it the temperature has no effect on particle size as shown in Fig 3a. No aggregation of particles was observed as in the previous study ${ }^{21}$.

At $\mathrm{pH} 8.0$ the particles exist in fully swollen state due to strong repulsive forces inside the network. These forces shifted the VPTT to such an extent that no transition in size was observed for all the prepared samples in the entire study range as indicated in Fig $3 b$.

\section{$3.3 U$ U-Visible spectroscopy}

Effect of $\mathrm{pH}$ and temperature on surface plasmon resonance (SPR) for poly(NIPAM-MAA)-Ag and poly(NIPAM-MAA)-Au was studied by UVVisible spectroscopy. The appearance of characteristic peaks at $525 \mathrm{~nm}$ in Fig $4 \mathrm{a}$ and at $400 \mathrm{~nm}$ in $4 \mathrm{~b}$, reveal the presence of Au and Ag NP's inside the microgel networks respectively. Our results indicate that increase in $\mathrm{pH}$ produce a clear blue shift and decrease in intensity in SPR peak. This shift is due to the inter-particle coupling, which changes with the size of polymer microgels. At $\mathrm{pH} 8.20$, the microgel particles are in swollen state and hold the metal NP's inside the network with high space among them, but with the decrease in $\mathrm{pH}$ to 3.20 , the microgel particles become shrink and inter-particle coupling of metal NP's takes place, which shift the SPR peak to high wavelength. The same trend was observed for both poly(NIPAM-MAA)-Au and poly(NIPAM-MAA)-Ag hybrid microgels.

Effect of temperature on SPR for poly(NIPAM-MAA)-Au and poly(NIPAM-MAA)-Ag were studied at $\mathrm{pH} 3.14$ and 8.36 in the temperature range from 20 to $50^{\circ} \mathrm{C}$. At $\mathrm{pH} 3.14$, it was found that increase in temperature produces red shift in SPR peak; this is due to the shrinkage of microgel particles, which brings the metal particles close to each other. At pH 8.36, no effect of temperature on SPR was observed due to the strong repulsive forces inside the microgel network, which inhibit the metal particles coupling; results obtained for poly(NIPAM-MAA)-Au are explored in Fig 5. The UV-Vis spectroscopic results strongly support the results obtained from DLS study for poly(NIPAMMAA). 

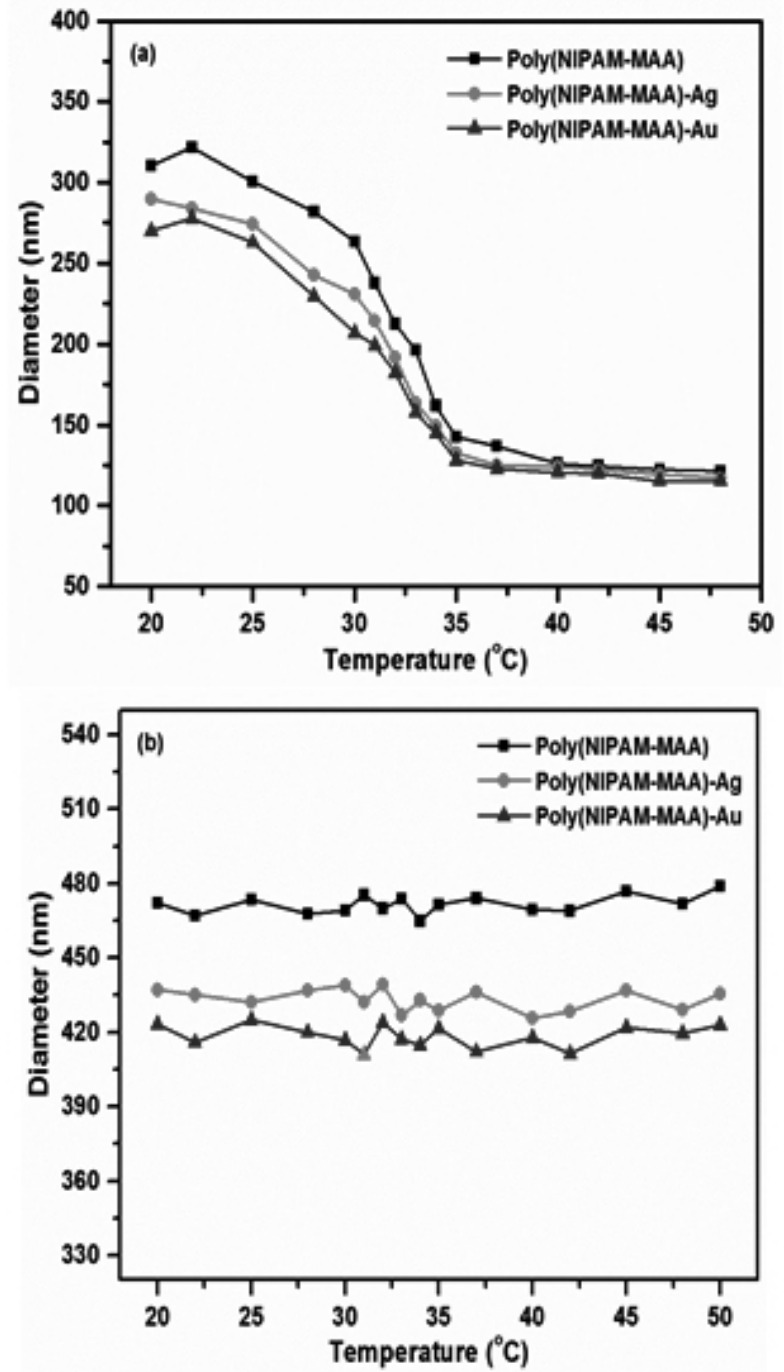

Fig 3 Hydrodynamic diameter as a function of temperature at (a) $\mathrm{pH} 3.11$ and (b) 8.0

\subsection{Transmission Electron Microscopy (TEM)}

Typical TEM images of hybrid poly(NIPAM-MAA)-Au and poly(NIPAMMAA)-Ag microgels are given in Fig 6. It is clear from the TEM results that metal particles are entrapped inside the polymer network successfully. The average size of Au NP's was found $\sim 12 \mathrm{~nm}$ while that of Ag NP's was $\sim 28$ $\mathrm{nm}$ by software Gatan Digitalmicrograph. The Ag NP's were found very close to each other, because the samples pH was kept 3.2 and were dried at high temperature before analysis.

\subsection{Catalytic activity of hybrid microgels}

The catalytic activity of poly(NIPAM-MAA)-Au and poly(NIPAMMAA)-Ag was investigated for the reduction of 4-nitrophenol (4-NP) to 4-aminophenol (4-AP) using excess sodium borohydride as a model reaction. The pure light yellow solution of 4-NP gives a characteristic peak at $318 \mathrm{~nm}$, addition of $\mathrm{NaBH}_{4}$ produced dark yellow solution by the formation of stable $p$-nitrophenolate ion, which shifted the peak to $400 \mathrm{~nm}{ }^{22}$. The peak intensity was almost constant for a few days, which clearly indicates that reaction cannot proceed without a catalyst. The addition of small amount of hybrid microgels caused the decrease in peak intensity with the passage of time and the yellow solution change to transparent. The reduction was further confirmed by the appearance of a new peak at $300 \mathrm{~nm}$ which is a characteristic peak for 4-AP ${ }^{23}$. The kinetics of this reaction was monitored by measuring the UV-Visible spectra as a function of time. Fig 7 shows the UV-Visible spectra obtained for poly(NIPAM-MAA)-Ag.
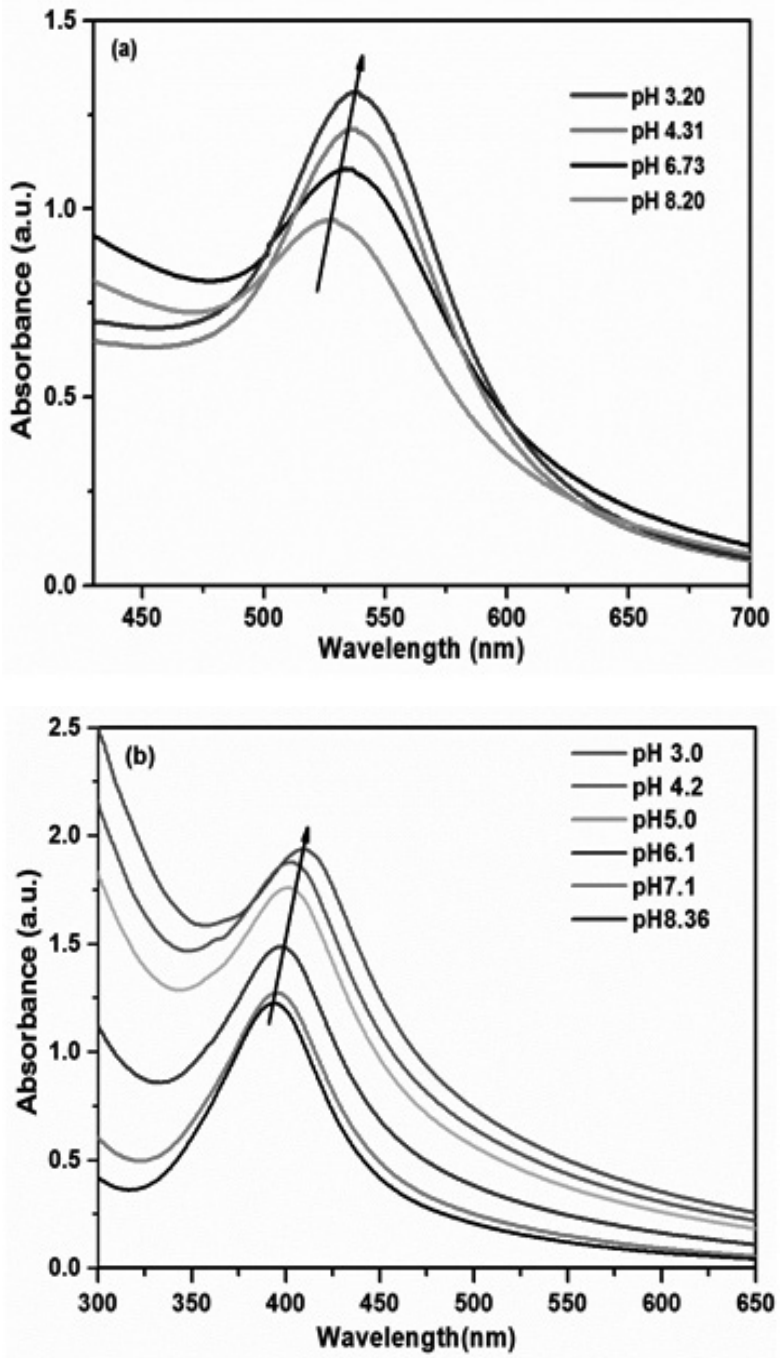

Fig $4 \mathrm{pH}$ effect on SPR for (a) poly(NIPAM-MAA)-Au and (b) poly(NIPAM-MAA)-Ag hybrid microgels

The reaction followed pseudo first order kinetics, because the concentration of $\mathrm{NaBH}_{4}$ was very high as compared to 4-NP, and could not affect the rate of reaction. The apparent rate constant $\left(k_{\text {app }}\right)$ was calculated from slope by plotting $\ln \left(\mathrm{C}_{\mathrm{t}} / \mathrm{C}_{\mathrm{o}}\right)$ vs time, as given in Fig 8 . The ratio of concentration of 4-NP $\left(\mathrm{C}_{\mathrm{t}}\right)$ at any time $\mathrm{t}$, to its initial concentration $\left(\mathrm{C}_{\mathrm{o}}\right)$ at $\mathrm{t}=0\left(\mathrm{C}_{\mathrm{t}} / \mathrm{C}_{\mathrm{o}}\right)$, can be directly obtained by the ratio of the respective absorbance $\left(\mathrm{A}_{\mathrm{t}} / \mathrm{A}_{\mathrm{o}}\right)$. The $k_{\text {app }}$ for poly(NIPAM-MAA)-Ag was found to be $1.62 \times \mathbf{1 0}^{-3} \mathrm{sec}^{-1}$, which is less than for poly(NIPAM-MAA)-Au $2.1 \times 10^{-3} \mathrm{sec}^{-1}$. This enhanced catalytic activity is due to the small size of Au NP's as compared to Ag NP's.

\section{CONCLUSION}

The poly(NIPAM-MAA) microgel was synthesized using DHEBA as cross-linker and was used as a microreactor for gold and silver nanoparticles. Temperature and $\mathrm{pH}$ sensitivity was checked by DLS and UV-Visible spectroscopy. The size and entrapment of metal NP's was confirmed by TEM. The hydrodynamic diameter of microgels showed inverse behavior to temperature while directly proportional to $\mathrm{pH}$. The surface plasmon resonance peaks for both poly(NIPAM-MAA)-Au and poly(NIPAM-MAA)-Ag showed red shift with increase in temperature and decrease of $\mathrm{pH}$. The catalytic activity of hybrid microgels were checked for the reduction of 4-nitro phenol to 4-aminophenol. Poly (NIPAM-MAA)-Au was found more efficient than poly(NIPAM-MAA)-Ag. The synthesis process is very convenient and can be used for large scale production. The catalysts can be recycled easily by centrifugation and can be applied for the reduction of other organic compounds. 

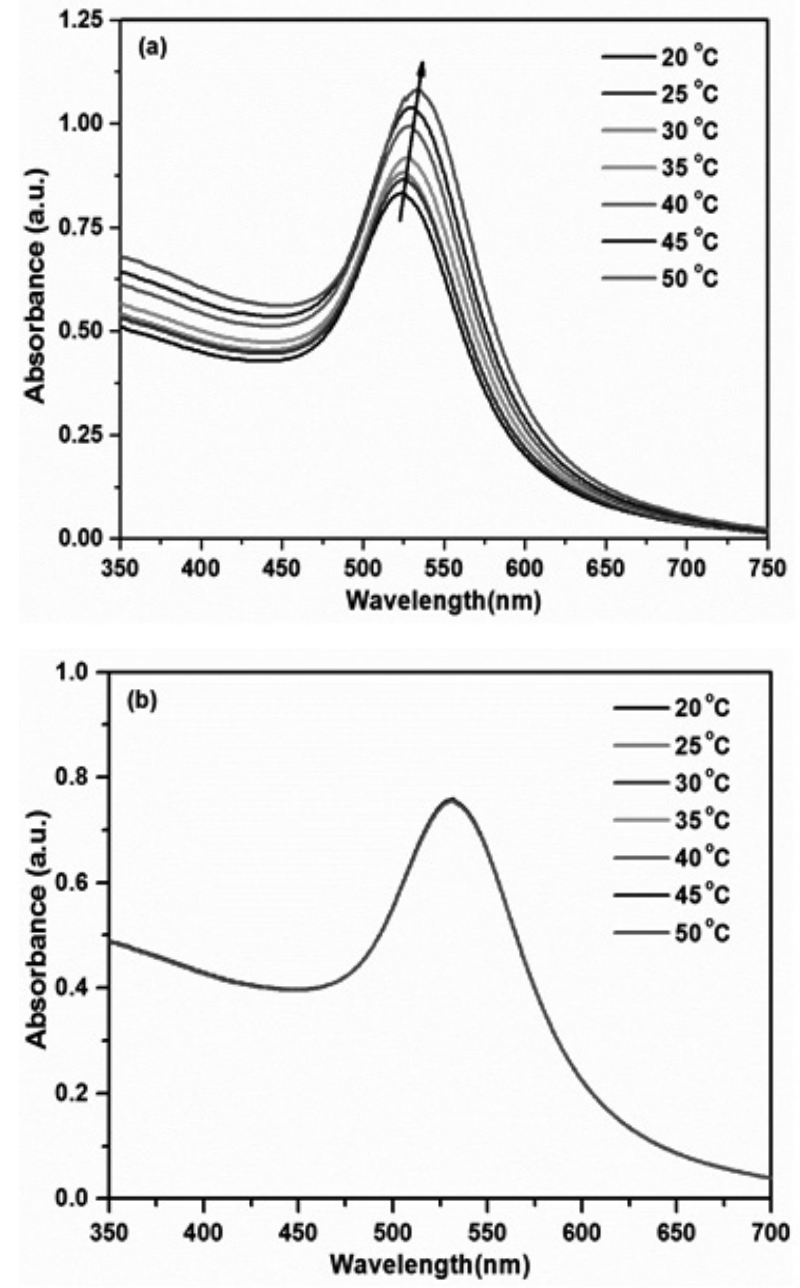

Fig 5 Temperature effect on SPR for poly(NIPAM-MAA)-Au at (a) $\mathrm{pH}$ 3.14 and (b) $\mathrm{pH} 8.36$

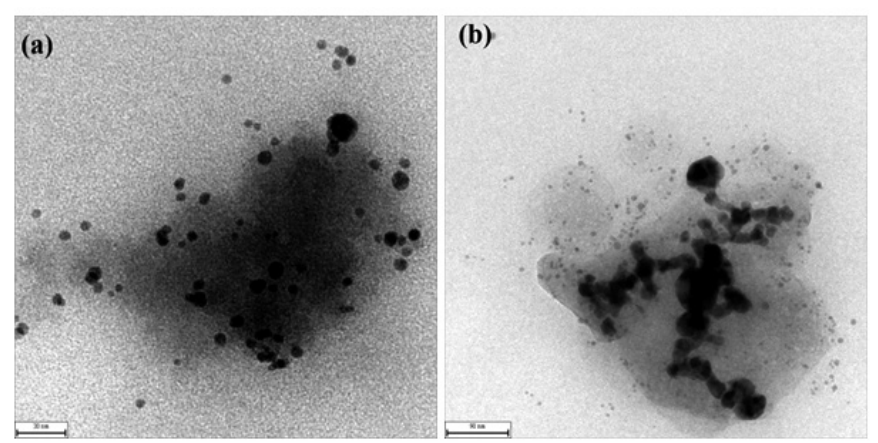

Fig 6 TEM images for (a) poly(NIPAM-MAA)-Au and (b) poly(NIPAMMAA)-Ag

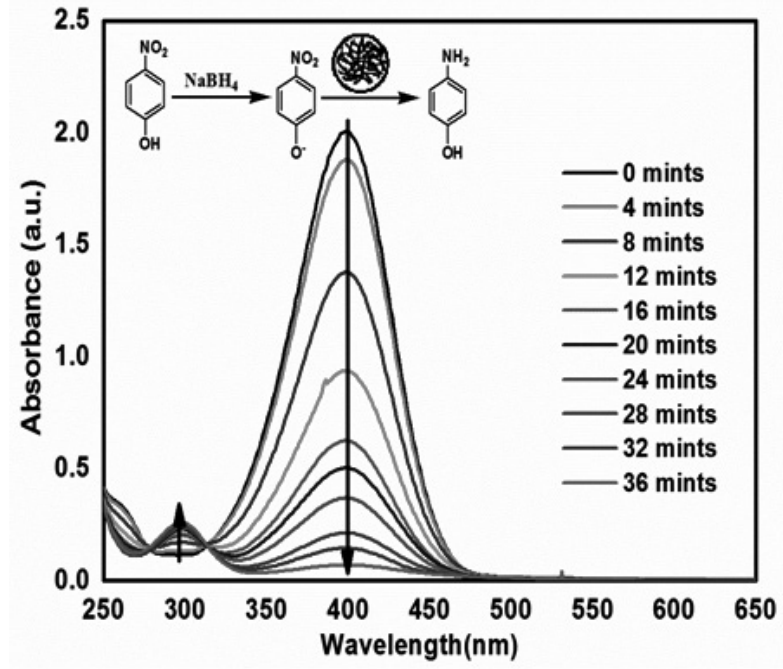

Fig 7 UV-vis spectra for the reduction of $p$-NP to $p$-AP by poly(NIPAMMAA)-Ag

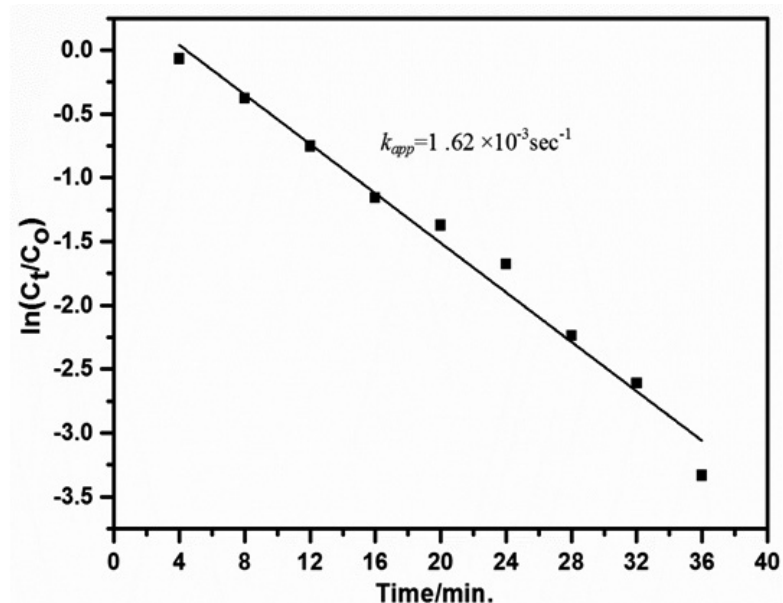

Fig 8 Plot of $\ln \left(\mathrm{C}_{\mathrm{t}} / \mathrm{C}_{\mathrm{o}}\right)$ vs. time for the conversion of $p$-nitrophenol by poly(NIPAM-MAA)-Ag

\section{ACKNOWLEDGMENTS}

"Dr. Luqman Ali Shah is highly grateful for financial support from Higher Education commission (HEC) of Pakistan for research project No:21-718/ SRGP/R\&D/HEC/2016".

\section{REFERENCES}

1. Kim, H.-Y.; Rho, W.-Y.; Lee, H. Y.; Park, Y. S.; Suh, J. S., Aggregation effect of silver nanoparticles on the energy conversion efficiency of the surface plasmon-enhanced dye-sensitized solar cells. Solar Energy 2014, 109, 61-69.

2. Ma, L.; Kohli, M.; Smith, A., Nanoparticles for combination drug therapy. ACS nano 2013, 7 (11), 9518-9525.

3. Han, D.-M.; Zhang, Q. M.; Serpe, M. J., Poly (N-isopropylacrylamide)co-(acrylic acid) microgel/Ag nanoparticle hybrids for the colorimetric sensing of $\mathrm{H} 2 \mathrm{O}$ 2. Nanoscale 2015, 7 (6), 2784-2789.

4. Herves, P.; Pérez-Lorenzo, M.; Liz-Marzán, L. M.; Dzubiella, J.; Lu, Y.; Ballauff, M., Catalysis by metallic nanoparticles in aqueous solution: model reactions. Chemical Society Reviews 2012, 41 (17), 5577-5587.

5. Yao, T.; Cui, T.; Wang, H.; Xu, L.; Cui, F.; Wu, J., A simple way to prepare $\mathrm{Au} @$ polypyrrole/Fe $3 \mathrm{O} 4$ hollow capsules with high stability and their application in catalytic reduction of methylene blue dye. Nanoscale 2014, 6 (13), 7666-7674

6. Bumajdad, A.; Ali, S.; Mathew, A., Characterization of iron hydroxide/ 
oxide nanoparticles prepared in microemulsions stabilized with cationic/ non-ionic surfactant mixtures. Journal of colloid and interface science 2011, 355 (2), 282-292.

7. Bakshi, M. S., Colloidal micelles of block copolymers as nanoreactors, templates for gold nanoparticles, and vehicles for biomedical applications. Advances in colloid and interface science 2014, 213, 1-20.

8. Wen, F.; Zhang, W.; Wei, G.; Wang, Y.; Zhang, J.; Zhang, M.; Shi, L., Synthesis of noble metal nanoparticles embedded in the shell layer of core- shell poly (styrene-co-4-vinylpyridine) micospheres and their application in catalysis. Chemistry of Materials 2008, 20 (6), 2144-2150.

9. Wu, H.; Liu, Z.; Wang, X.; Zhao, B.; Zhang, J.; Li, C., Preparation of hollow capsule-stabilized gold nanoparticles through the encapsulation of the dendrimer. Journal of colloid and interface science 2006, 302 (1), 142148.

10. Wunder, S.; Polzer, F.; Lu, Y.; Mei, Y.; Ballauff, M., Kinetic analysis of catalytic reduction of 4-nitrophenol by metallic nanoparticles immobilized in spherical polyelectrolyte brushes. The Journal of Physical Chemistry C 2010, 114 (19), 8814-8820.

11. Kumacheva, E., Hydrogels: the catalytic curtsey. Nature materials $\mathbf{2 0 1 2}$ $11(8), 665-666$.

12. Dietrich, S.; Schulze, S.; Hietschold, M.; Lang, H., Au nanoparticles stabilised by PEGylated low generation PAMAM dendrimers: Design, characterisation and properties. Journal of colloid and interface science 2011, 359 (2), 454-460.

13. Agrawal, G.; Schürings, M. P.; van Rijn, P.; Pich, A., Formation of catalytically active gold-polymer microgel hybrids via a controlled in situ reductive process. Journal of Materials Chemistry A 2013, 1 (42), 1324413251.

14. Zheng, Y.; Wang, A., Ag nanoparticle-entrapped hydrogel as promising material for catalytic reduction of organic dyes. Journal of Materials
Chemistry 2012, 22 (32), 16552-16559.

15. Shah, L. A.; Farooqi, Z. H.; Naeem, H.; Shah, S. M.; Siddiq, M., Synthesis and Characterization of Poly ( $\mathrm{N}$-isopropylacrylamide) Hybrid Microgels with different Cross-linker Contents. JOURNAL OF THE CHEMICAL SOCIETY OF PAKISTAN 2013, 35 (6), 1522-1529.

16. Shah, L. A.; Chen, W.; Siddiq, M.; Hu, J.; Dong, A.; Yang, D., Thermal and $\mathrm{pH}$ Dual Responsive Copolymer and Silver Nanoparticle Composite for Catalytic Application. Chinese Journal of Chemistry 2015, 33 (4), 467472 .

17. Hu, X.; Tong, Z.; Lyon, L. A., Control of poly (N-isopropylacrylamide) microgel network structure by precipitation polymerization near the lower critical solution temperature. Langmuir 2011, 27 (7), 4142-4148.

18. Kim, M.; Kim, J.; Lee, H.; Kim, J.; Yang, J., Colloid Surf B 2005, 46, 57. CrossRef, PubMed, CAS, Web of Science ${ }^{\circledR}$ Times Cited 32.

19. Agrawal, G.; Schürings, M.; Zhu, X.; Pich, A., Microgel/SiO 2 hybrid colloids prepared using a water soluble silica precursor. Polymer 2012, 53 (6), 1189-1197.

20. Bhattacharya, S.; Eckert, F.; Boyko, V.; Pich, A., Temperature $\square$, pH $\square$, and Magnetic $\square$ Field $\square$ Sensitive Hybrid Microgels. Small 2007, 3 (4), 650657.

21. Naeem, H.; Farooqi, Z. H.; Shah, L. A.; Siddiq, M., Synthesis and characterization of $\mathrm{p}$ (NIPAM-AA-AAm) microgels for tuning of optical Properties of silver nanoparticles. Journal of Polymer Research 2012, 19 (9), 1-10.

22. Huang, J.; Vongehr, S.; Tang, S.; Lu, H.; Meng, X., Highly catalytic PdAg bimetallic dendrites. The Journal of Physical Chemistry C 2010, 114 (35), 15005-15010.

23. Tang, S.; Vongehr, S.; He, G.; Chen, L.; Meng, X., Highly catalytic spherical carbon nanocomposites allowing tunable activity via controllable $\mathrm{Au}-\mathrm{Pd}$ doping. Journal of colloid and interface science 2012, 375 (1), 125-133. 